\title{
Efficacite D'un Appât Proteinique Alimentaire «Great Fruit Fly Bait (GFFB)» Dans la Lutte Contre Les Mouches des Fruits en Culture de Manguier
}

\author{
Minhibo Yves Magloire, \\ Chercheur, Centre National de Recherche Agronomique, \\ Station de Korhogo-Lataha, Côte d'Ivoire
}

Akpesse Akpa Alexandre Moüse,

Maitre de conférences, UFR-Biosciences, Université Felix Houphouet

Boigny de Cocody, Laboratoire de Zoologie et de Biologie Animale,

Côte d'Ivoire

\section{Diby Yao Kan Séraphin,}

Docteur en entomologie agricole, UFR-Biosciences, Université Felix Houphouet Boigny de Cocody, Laboratoire de Zoologie et de Biologie

Animale, Côte d'Ivoire

\section{Hala N'klo,}

Directeur de recherches, Centre National de Recherche Agronomique, Station de recherche Marc DELORME, Côte d'Ivoire

\section{Koua K. Hervé,}

Professeur Titulaire, UFR-Biosciences, Université Felix Houphouet Boigny de Cocody, Laboratoire de Zoologie et de Biologie Animale, Côte d'Ivoire

Doi:10.19044/esj.2019.v15n27p111 URL:http://dx.doi.org/10.19044/esj.2019.v15n27p111

\section{Resume}

Les mouches des fruits constituent de redoutables ravageurs de mangues et d'autres fruits en Côte d'Ivoire. Afin de lutter efficacement contre les mouches des fruits, un test d'évaluation de l'efficacité de l'appât alimentaire Great Fruit Fly Bait (GFFB) contenant la matière active, l'Abamectine, a été effectué dans trois vergers du département d'Odienné. Le témoin de référence a été le Success Appat (GF-120) ajouté à un témoin non traité. Le traitement a été effectué sur l'ensemble de la parcelle élémentaire une fois par semaine pendant sept semaines. Le traitement a consisté à pulvériser sur le feuillage de chaque arbre traité (les fruits n'ont pas subi de traitement). Après sept semaines d'application, 100 mangues ont été récoltées de manière aléatoire sur chaque parcelle élémentaire. Celles qui sont piquées ont été comptées et incubées au laboratoire jusqu'à l'émergence des mouches adultes qui ont été ensuite identifiées. Les résultats obtenus sur les trois (3) 
vergers montrent que le niveau d'infestation des mangues a été faible dans les vergers traités aux GFFB (0,45 pupe par fruit et par poids pour la dose 1 Litre/ha et 0,04 pupe par fruit et par poids pour la dose de 1,5 Litre/ha) et GF120 (0,36 pupe par fruit et par poids). Par contre dans les parcelles non traitées, les niveaux d'infestations ont été élevés (12,47 pupes par fruit et par poids). En outre, les pourcentages de protection ont été respectivement de $81,78 \% ; 98,38 \%$ et $85,42 \%$ respectivement pour GFFB à la dose de $1 \mathrm{~L} / \mathrm{ha}$, 1,5 L/ha et GF120. l'utilisation du produit testé GFFB tout comme le produit de référence GF120, a permis de protéger efficacement les fruits sur les vergers de manguiers.

Mots clés: Great Fruit Fly Bait, lutte biologique, Tephritidae, Fruit, Côte d'Ivoire 


\title{
Effectiveness of a "Great Fruit Fly Bait" (GFFB) in the Control of Fruit Flies in Mango Cultivation
}

\author{
Minhibo Yves Magloire, \\ Chercheur, Centre National de Recherche Agronomique, \\ Station de Korhogo-Lataha, Côte d'Ivoire \\ Akpesse Akpa Alexandre Moïse,
}

Maitre de conférences, UFR-Biosciences, Université Felix Houphouet

Boigny de Cocody, Laboratoire de Zoologie et de Biologie Animale,

Côte d'Ivoire

Diby Yao Kan Séraphin,

Docteur en entomologie agricole, UFR-Biosciences, Université Felix Houphouet Boigny de Cocody, Laboratoire de Zoologie et de Biologie Animale, Côte d'Ivoire

\section{Hala N'klo,}

Directeur de recherches, Centre National de Recherche Agronomique,

Station de recherche Marc DELORME, Côte d'Ivoire

Koua K. Hervé,

Professeur Titulaire, UFR-Biosciences, Université Felix Houphouet Boigny de Cocody, Laboratoire de Zoologie et de Biologie Animale, Côte d'Ivoire

\begin{abstract}
Fruit flies are a major pest of mangoes and other fruits in Côte d'Ivoire. In order to effectively control fruit flies, a test to evaluate the effectiveness of Great Fruit Fly Bait (GFFB) containing the active ingredient, Abamectin, was carried out in three orchards in the Odienne department. The reference control was the Success Appat (GF-120) added to an untreated control. The treatment was carried out on the entire elementary plot once a week for seven weeks. The treatment consisted of spraying the foliage of each treated tree (the fruits were not treated). After seven weeks of application, 100 mangoes were randomly harvested from each elementary plot. Those that are bitten were counted and incubated in the laboratory until the emergence of adult flies that were then identified. The results obtained on the three (3) orchards show that the level of mango infestation was low in orchards treated with GFFB $(0.45$ pupa per fruit per weight for the $1 \mathrm{Litre} / \mathrm{ha}$ dose and 0.04 pupa per fruit per weight for the 1.5 Litre/ha dose) and GF120 ( 0.36 pupe per fruit per weight). In contrast, in untreated plots, infestation levels were high (12.47 pupae per
\end{abstract}


fruit per weight). In addition, the protection percentages were $81.78 \%$ and $98.38 \%$ and $85.42 \%$ respectively for GFFB at $1 \mathrm{~L} / \mathrm{ha}, 1.5 \mathrm{~L} / \mathrm{ha}$ and $\mathrm{GF} 120$ respectively. The use of the GFFB test product as well as the GF120 reference product effectively protected the fruits on mango orchards.

Keywords: Great Fruit Fly Bait, Biological control, Tephritidae, Fruit, Côte d'ivoire

\section{Introduction}

Les fruits jouent un rôle économique et social prépondérant en subvenant aux besoins des populations (Mamadou, et al., 2003). En Côte d'Ivoire, la mangue (Mangifera indica L.) récoltée au Nord du pays représente le troisième produit de rente après le coton (Gossypium hirsutum L.) et l'anacarde (Anacardium occidentale L.) (N'Dépo, et al., 2009). Pendant la campagne 2016 de la mangue, la production a été estimé à $100.000 \mathrm{~T}$, dont 32.628 T ont été exportées (contre 22.769 T en 2015), ce qui a permis à la Côte d'Ivoire d'être le ler exportateur de mangue en Afrique et troisième fournisseur sur le marché Européen après le brésil et le Pérou (AIP, 2016). La mangue représente une source fondamentale de nutrition dans les régions soudano sahéliennes de l'Afrique de l'Ouest et est riche en potassium, alpha carotène, vitamine $\mathrm{C}$ et calcium (Vayssières et al., 2008).

Les mouches des fruits (Tephritidae) avec plus de 250 espèces économiquement nuisibles (White et Elson-Harris, 1992), constituent un des problèmes entomologiques majeurs des mangues en Afrique de l'Ouest et dans le monde (Mwatawala et al., 2004 ; Rey et al., 2004 ; Vayssières et al., 2004 et 2005 ; Anonymes, 2006). Elles s'attaquent à plus de 50 espèces fruitières dont les agrumes et les baies (Oukil et al., 2002 ; Vasquez et al., 2002). Les mouches piquent essentiellement les fruits en pré-maturité ou en maturité (Quilici et al., 2005). Elles pondent leurs œufs dans les fruits à faible profondeur. Les larves issues de ces œufs (vers ou asticots), se nourrissent de la pulpe du fruit en creusant des galeries. Des pourritures secondaires se développent dans les fruits soumis à des piqûres de pontes et chutent (Bayart et al., 1997 ; Barbet, 2000 ; Rey et al.,2004 ; Quilici et al., 2006). Ainsi, les chutes et les pourritures sont-elles responsables de la perte de nombreux fruits. Les dégâts, en Côte d'Ivoire sont estimé à 17\% en début de campagne ; 69\% en milieu de campagne et $80 \%$ en fin de campagne, voire $100 \%$ s'il n'y pas de mesure de protection des vergers (N'Dépo et al., 2010).

Les méthodes de lutte en vigueur sont la lutte biologique avec les parasitoïdes, les fourmis tisserandes, le piégeage avec les attractifs sexuels et les appâts alimentaires. Dans la recherche des produits d'alternance, un test d'efficacité de l'appât alimentaire Great Fruit Fly Bait (GFFB) contre les mouches des fruits en culture de manguiers a été initié. Pour atteindre cet 
objectif, des traitements par taches ont été effectués sur les feuillages des manguiers pour comparer l'efficacité des produits testés à la variante GF120.

\section{Matériel et Méthodes}

\section{Zone d'étude}

L'expérimentation a eu lieu sur trois sites du département d'Odienné, zone Nord-Ouest de la Côte d'Ivoire, situé à l'Est du massif du Denguélé. Les coordonnées des sites sont les suivants: site $1 \mathrm{du}$ verger 1 (Latitude 09³1.870’ N, Longitude: 007³4.330’W), site 2 du verger 2 (Latitude $09^{\circ} 34.257^{\prime} \mathrm{N}$, Longitude $007^{\circ} 32.276^{\prime} \mathrm{W}$ ) et site $3 \mathrm{du}$ verger 3 (Latitude $09^{\circ} 30.306^{\prime} \mathrm{N}$, Longitude $\left.007^{\circ} 33.859^{\prime} \mathrm{W}\right)$. Le type de végétation est la savane. Les sols sont de types ferralitiques. Le $\mathrm{pH}$ varie entre 4,5 et 6. Le climat est du type Soudanais. Il est très chaud et très sec, avec un vent sec appelé l'Harmattan de décembre à janvier. La grande saison sèche (octobre - mai) précède la saison des pluies marquée par deux maxima dont l'un en juin et l'autre en septembre. Les précipitations moyennes varient entre $83 \mathrm{~mm}$ et 299 mm. Les températures moyennes varient entre 25 et $29,1^{\circ} \mathrm{C}$. L'étude a été conduite dans trois vergers qui étaient composées de la variété de mangues Kent.

\section{Matériel}

\section{Matériel végétal}

Le matériel végétal était constitué de pieds de manguiers (Mangifera indica L., famille des anacardiacées) de la variété Kent.

\section{Matériel animal}

Le matériel animal était constitué des mouches des fruits adultes capturés dans les vergers.

\section{Matériel technique}

Le matériel technique est composé d'un pulvérisateur à dos de 20 litres; du produit commercial à tester, Great @ Fruit Fly Bait (GFFB), contenant un appât liquide à base de protéine hydrolysée et une matière active, l'Abamectine à la concentration de $0,1 \%$; du produit de référence ou témoin positif, Success appât (GF 120), contenant un mélange d'appât liquide ainsi que la matière active Spinosad à la concentration de $0,24 \mathrm{~g}$ m.a./L. Des pièges à Para-phéromones sexuels de synthèse, imitant une phéromone produite naturellement par les femelles, ont été utilisés. Il s'agit: du Methyl Eugenol qui attire les mâles du genre Bactrocera et du Terpinyl acétate qui attire les mâles du genre Ceratitis.

Des piluliers et de l'alcool à $70^{\circ}$ ont été utilisés pour la conservation des mouches. La graisse de moteurs a été mise sur les branches pour empêcher 
toute activité de prédation d'Oecophyla longinoda. Un GPS de marque Garmin a permis de déterminer les références géographiques des sites.

\section{Méthodes}

\section{Dispositif expérimental}

Le dispositif expérimental est un bloc de Fischer comprenant 4 traitements et 3 répétitions entièrement randomisés :

1. T0 : verger témoin non traité

2. T1 : GFFB à la dose de $1 \mathrm{~L} / \mathrm{ha}$;

3. T2 : GFFB à la dose de $1,5 \mathrm{~L} / \mathrm{ha}$;

4. T3 : GF-120 à la dose de $1 \mathrm{~L} /$ ha ou produit de référence ou témoin positif.

Chaque verger est composé de 450 manguiers subdivisés en 3 parcelles élémentaires de 100 arbres. Tous les quatres traitements ont été mis en place dans chaque verger et repeté 3 fois.

\section{Inventaire des mouches}

Pour l'inventaire et le suivi des niveaux de pullulation des populations adultes de mouches, trois pièges ont été installés sur chaque parcelle élémentaire. Ce sont deux pièges à attractifs sexuels (Méthyl eugénol, Terpinyl acétate) et un piège à appât alimentaire (Torula).

\section{Évaluation de l'efficacité du GFFB}

Les applications ont été effectuées sur l'ensemble de la parcelle élémentaire une fois par semaine pendant sept semaines. L'application a consisté à pulvériser le feuillage de chaque arbre excepté les fruits. Après sept semaines d'application, 100 mangues non matures, choisis de manière aléatoire et pesées, ont été récoltées sur chaque parcelle élémentaire. Les mangues piquées ont été comptées et incubées au laboratoire jusqu'à l'émergence des mouches adultes qui ont été ensuite identifiées.

Le niveau d'infestation des fruits a été déterminé à partir du rapport entre le nombre de pupe émergé et le poids total mangues en $\mathrm{kg}$.

Le niveau d'infestation a été calculé selon la formule :

Niveau d'infestation $\left(\right.$ Pupes/kg) $==\frac{\text { Nombre de pupes collectées }}{\text { Poids total mangues en } \mathrm{kg}}$

Le pourcentage de protection du verger est déterminé par la différence entre le niveau d'infestation du témoin et du traité par rapport au niveau d'infestation du témoin multiplié par 100 (Billah et al., 2014)

Pourcentage protection $(\%)=\frac{((\mathrm{NT}-\mathrm{T}) / \mathrm{NT})}{100}$

$\mathbf{N T}=$ Non traité $\quad \mathbf{T}=$ Traité 


\section{Analyse des données}

Une analyse de la variance a été utilisée pour comparer les différents traitements. Le test de Student Newman-Keuls a été utilisé pour la séparation des moyennes au seuil de 5\%. Le logiciel SPSS (Statistique Package for Social Science) a été utilisé pour le traitement des données.

\section{Résultats}

\section{Inventaire des mouches}

Huit espèces de mouches des fruits ont été identifiées dans les 3 vergers de la localité d'Odienné à savoir : Bactrocera dorsalis, Bactrocera cucurbitae, Ceratitis cosyra, Ceratitis punctata, Ceratitis bremii, Ceratitis fasiventris, Dacus bivittatus et Dacus punctatifrons (Figure 1). L'espèce exotique $B$. dorsalis a été la plus dominante avec $99,41 \%$ de présence. Elle est suivie par les espèces $C$. cosyra et $C$. punctata (Figure 1).

Sur les 3 sites, le nombre moyen de mouches capturé par les pièges disposés dans les parcelles témoins, est supérieur à ceux des parcelles traitées aux appâts alimentaires. Au niveau des parcelles traitées, l'analyse statistique ne montre pas de différence significative entre les captures des mouches des parcelles traitées malgré que le traitement T2 présente moins de capture que les deux autres traitements (Figure 2).

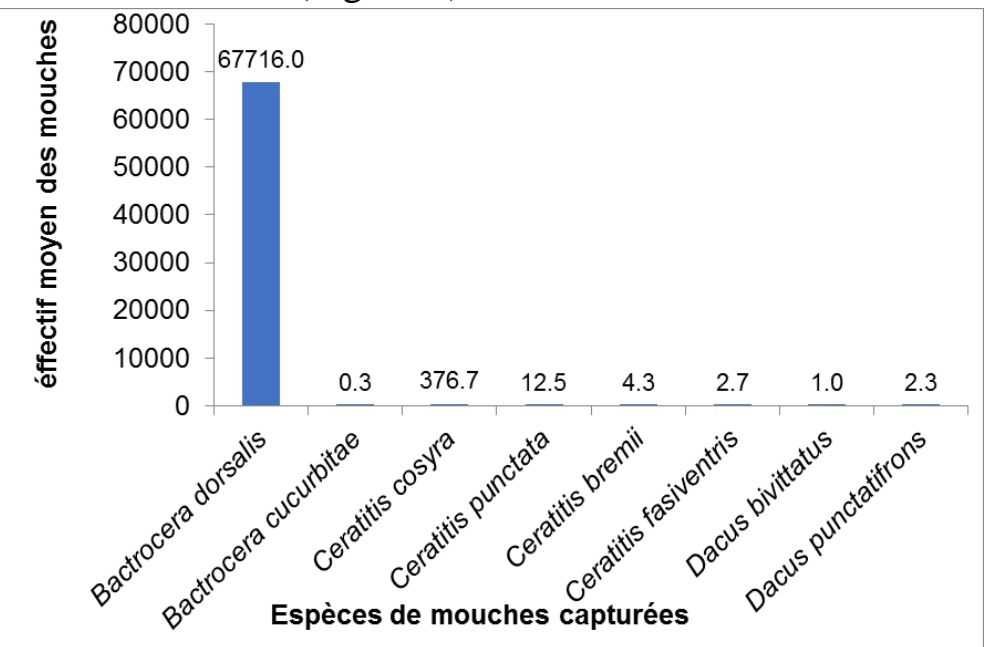

Figure 1: Effectif moyen des espèces de mouches de fruits dans la 


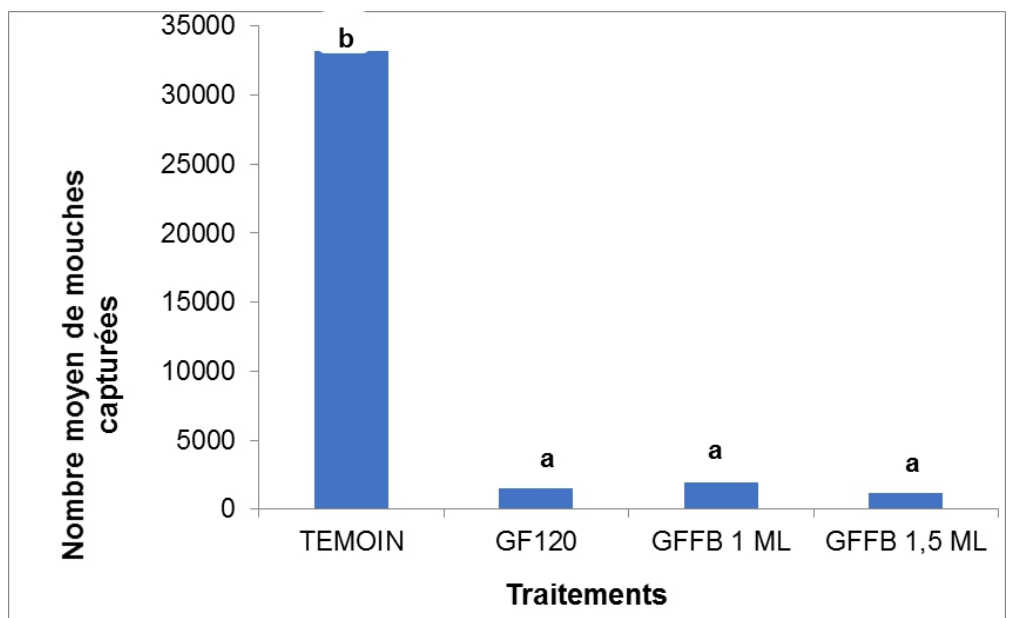

Figure 2: Effectif moyen de mouches de fruits en fonction des traitements dans la localité d'Odienné

Au niveau de la dynamique des mouches capturées dans les vergers traitées et non traitées, il est constaté une différence hautement significative des captures de mouches sur les trois sites étudiés $(\mathrm{p}=0,00005)$. Pour le verger 1 , de la $1^{\text {ère }}$ semaine à la $3^{\text {ème }}$ semaine de traitement, il est observé une hausse des captures au niveau des parcelles témoins. Par contre, une baisse significative des captures est observée au niveau des parcelles traitées (Figure 3). De la $4^{\text {ème }}$ semaine à la $7^{{ }^{\mathrm{ème}}}$ semaine, soit de juin à juillet, la population des mouches baisse de manière significative jusqu'à obtenir moins de 20 captures par verger. Par contre, pour cette même période, la population de mouches dans les vergers témoin décroit mais elle est toujours supérieure à celle des parcelles traitées (Figure 3).

Pour les vergers 2 et 3 , les fluctuations sont identiques à celle du verger 1. Au niveau des vergers 2 et 3 , la hausse des captures pour le verger témoin est observée respectivement de la $1^{\text {ème }}$ semaine à la $2^{\text {ème }}$ semaine de traitement et de la $1^{\text {ème }}$ semaine à la $5^{\text {ème }}$ semaine de traitement (Figures 4 et 5). Par contre, les captures au niveau des deux vergers baissent significativement de la $1^{\text {ème }}$ semaine à la $3^{\text {ème }}$ semaine de traitement pour le verger 2 et de la $1^{\text {ème }}$ semaine à la $2^{\text {ème }}$ semaine de traitement pour le verger 3 (Figures 4 et 5). L'analyse statistique ne montre pas de différence significative au niveau des vergers traités à l'appât alimentaire $(\mathrm{P}=0,745)$. 


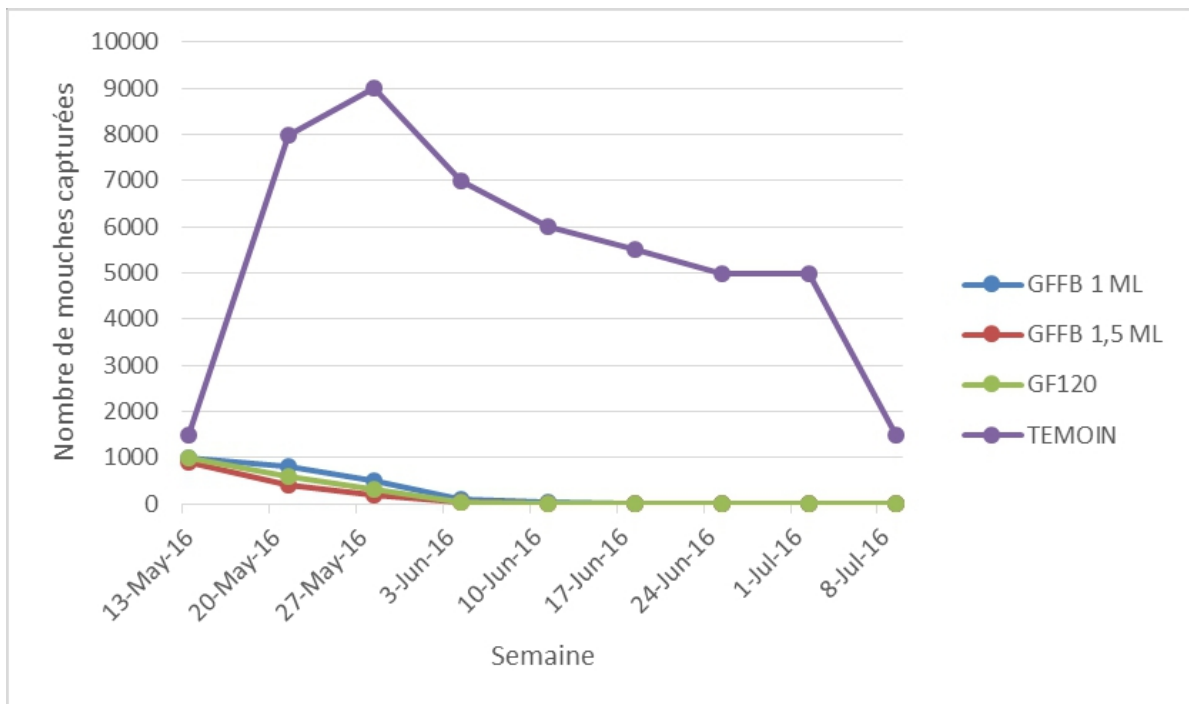

Figure 3: Fluctuation des populations de mouches de fruits dans le verger 1

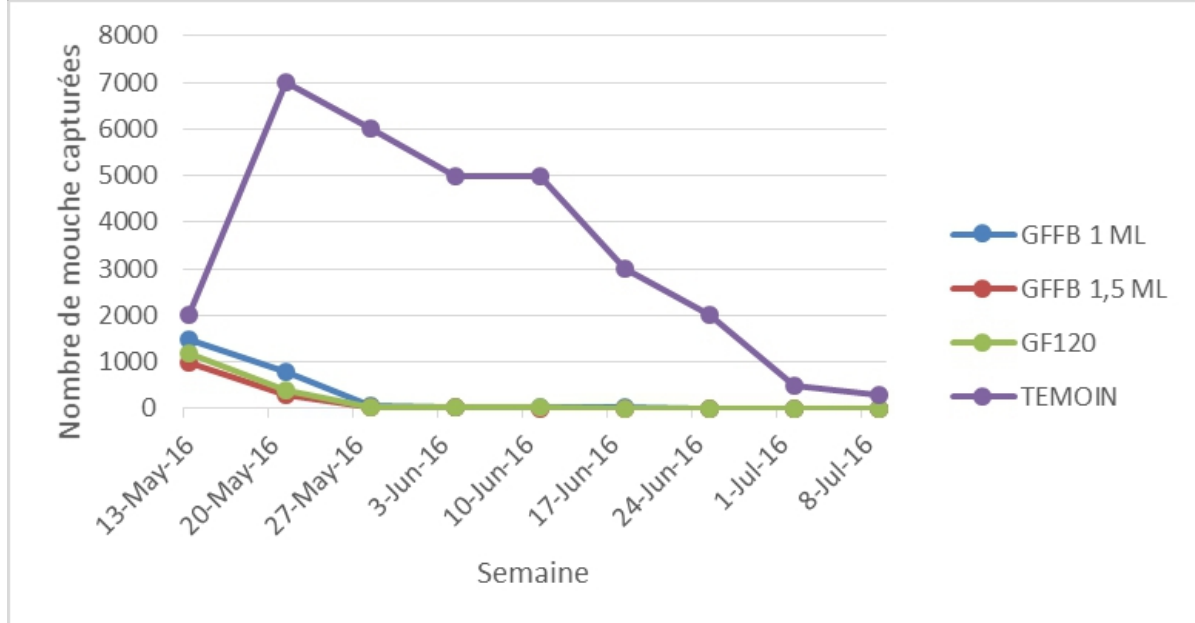

Figure 4 : Fluctuation des populations de mouches de fruits dans le verger 2

\section{Évaluation de l'efficacité des appâts alimentaires}

Les résultats obtenus sur les trois vergers montrent que le niveau d'infestation des mangues est faible après traitement des vergers par rapport au verger témoin. Après traitement au GFFB, il est observé 0,45 pupe par fruit et par poids pour le traitement T1, 0,04 pupe par fruit et par poids pour le traitement T2 et 0,36 pupe par fruit et par poids pour le traitement T3. Par contre, dans les parcelles non traitées (T0), les niveaux d'infestations ont été élevés (12,47 pupes par fruit et par poids). 


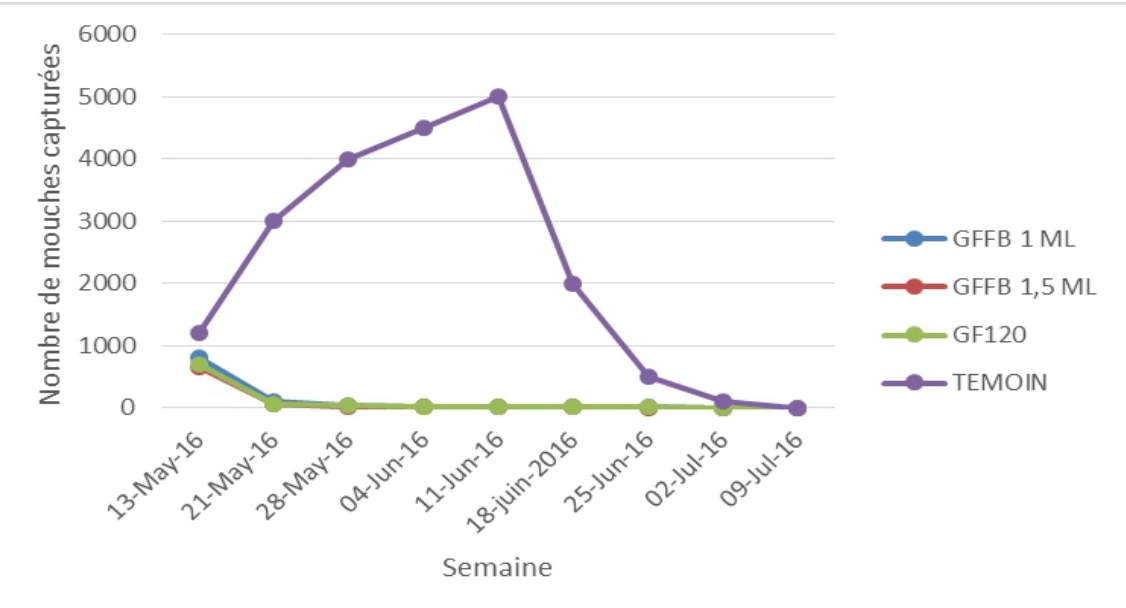

Figure 5: Fluctuation des populations de mouches de fruits dans le verger 3

Les pourcentages de protection des vergers ont été de $81,78 \% ; 98,38 \%$ et $85,42 \%$ respectivement pour GFFB à la dose de 1L/ha (T1), GFFB à la dose de 1,5L/ha (T2) et pour GF120 (T3). Par ailleurs, il n'existe pas de différence significative entre les pourcentages de protections des vergers traités $(\mathrm{P}=$ 0,596 ). Par contre, il y a eu plus de larves de mouches émergées des échantillons témoins par rapport aux échantillons des produits testés (Figure $6)$.

Figure 6: Taux moyen des fruits piqués par traitement

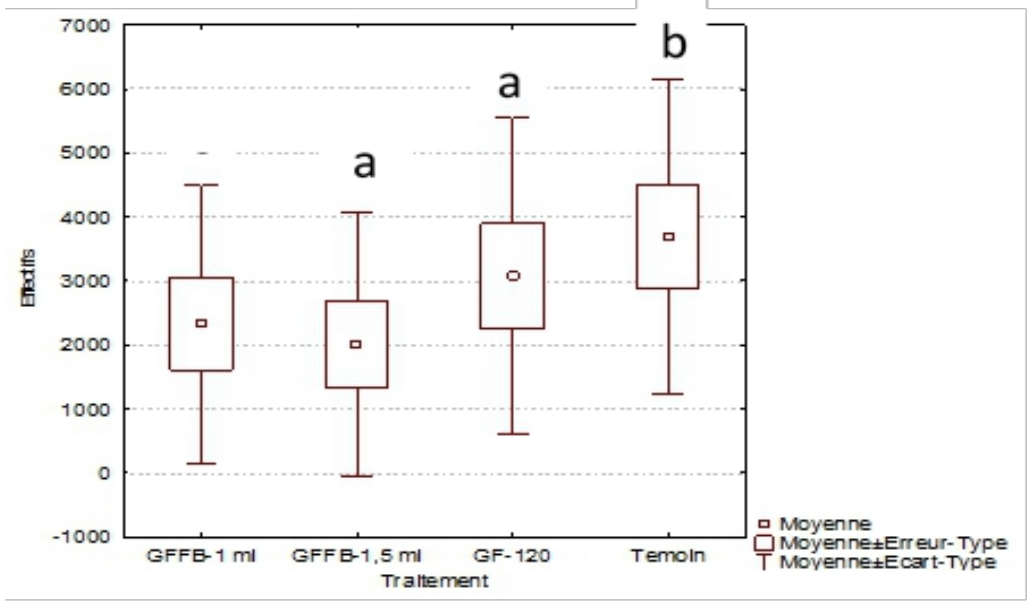

\section{Discussion}

Les pièges installés ont permis de capturer huit espèces de mouche de fruit. Leur présence sur les manguiers ont été déjà signalées dans plusieurs régions d'Afrique (Vayssières et al., 2004 et 2005 ; Mwatawala et al., 2004 et 2006) et en Côte-d'Ivoire (N'dépo, 2006 ; N'Dépo et al., 2009). Il a été constaté une dominance de l'espèce $B$. dorsalis sur l'ensemble des vergers. La 
forte capture de cette espèce serait probablement due à l'effet attractif du méthyl eugenol. En effet, le méthyl eugénol est très attractifs et possède un large spectre d'action autour d'un piège, fournissant ainsi des informations sur l'abondance de B. dorsalis sur une grande distance (Mwatawala et al., 2006, Vayssières et al., 2007). La hausse des captures de $B$. dorsalis dans les vergers est aussi imputable au cycle biologique naturel de l'insecte dont les niveaux de populations augmentent avec l'arrivée de la saison des pluies (White et Elson-Harris, 1992). Par ailleurs, les pièges créent des épicentres d'attraction pour les mâles qui y convergent souvent à partir de longues distances (Vayssières et al., 2009). En outre, l'abondance des mouches observées dans les vergers témoins dans le mois de Mai ou dans les trois premières semaines serait due à la période de forte fructification et des premières pluies. La diminution des effectifs des mouches est probablement due au rythme de réduction progressive des quantités de fruits mûrs sur les arbres et des pluies. En ce qui concerne l'efficacité des traitements au produit GFFB, en comparaison avec le témoin non traité, les résultats montrent que l'utilisation du produit GFFB a permis de réduire de façon significative le nombre de larves dans les mangues et l'émergence des mouches par rapport au verger témoin. Les résultats obtenus montrent que le GFFB à la dose 1,5 $\mathrm{ml}$ assure une meilleure contrôle des populations des mouches des fruits avec un pourcentage de protection de $98,38 \%$ contre $85,42 \%$ pour le GF-120. Ce pourcentage est presque identique à celui obtenu lors des travaux de Billah et al., (2014) au Ghana où le pourcentage de protection avec le GFFB a été de $95,4 \%$ et celui du GF-120 de 80,7\%. Les travaux de N'Dépo et al., (2010), ont montré que l'application du GF 120 sur des manguiers en Côte d'ivoire présentait un taux de protection élevé et un niveau d'infestation faible. Toutefois, le fait qu'il n'y ai pas de différence significative de protection des vergers entre les différents produits, révèle l'efficacité de l'utilisation des appâts alimentaires dans la protection des vergers de manguiers.

Vayssières et al. (2009) ont aussi démontré dans leurs travaux, que l'application du Success Appat ${ }^{\circledR}$ dans les vergers de manguiers du Bénin, a permis d'obtenir une différence hautement significative entre les vergers non traités et les vergers traités $(16,97 \pm 1,96$ pupes $/ \mathrm{kg}$ de fruits pour les parcelles non traitées et 3,17 $\pm 0,61$ pupes/kg de fruit pour les parcelles traitées au Success Appat ${ }^{\circledR}$ en 2006 et 34,5 $\pm 3,53$ pupes $/ \mathrm{kg}$ de fruit pour les parcelles non traitées contre 3,67 $\pm 0,67$ pupes/kg de fruit pour les parcelles traitées en 2007).

Moins de pupes ont émergé des échantillons de fruits traités aux produits GFFB et GF 120, cela peut être dû à l'état des femelles qui ont piqués ces fruits ; ces femelles n'étaient certainement pas toutes fécondées. En outre, ces femelles non fécondées ont piqué les fruits sans y pondre les œufs. 


\section{Conclusion}

Au terme de cette étude, l'efficacité de l'utilisation du GFFB est avérée. Ces résultats sont encourageants car les traitements effectués ont eu une incidence sur le nombre de mouches capturées. Ainsi, l'utilisation du produit GFFB a permis d'avoir un faible niveau d'infestation des vergers sur les 3 sites. Après sept semaines d'applications, l'efficacité de l'appât alimentaire a été confirmée avec les pourcentages de protection élevés pour la dose 1,5 L/ha. Il apparaît judicieux de considérer la dose 1,5 L/ha comme étant la dose optimale dans cette étude. Un des avantages non négligeables de cette méthode, est qu'elle ne pollue pas l'environnement. Par ailleurs, les pulvérisations par tâche avec les appâts liquides, devront faire partie des stratégies de lutte intégrée contre les mouches de fruits.

\section{References:}

1. Anonyme (2006). Les Valeurs nutritionnelles de la mange. Canadian Entomologist, 96, 810-811.

2. AIP. (2016). Campagne de la filière mangue en Côte d'Ivoire. http://news.abidjan.net/h/602544.html. 15 Juin 2019.

3. Barbet A. (2000). Suivi des populations de Ceratitis spp (Diptera : Tephritidae) au nord Côte-d'Ivoire dans la perspective d'une lutte raisonnée en verger de manguiers. Mémoire d'Ingénieur des Techniques Agricoles, Montpellier, France, 52 p.

4. Bayart J. D., Phalip M., Régis L., Gueudré F. (1997). Mouches des fruits : Bilan de quatre années de contrôle des fruits à l'importation en Ille-de-France. Phytoma- La Défense des végétaux, 490 : 20-25.

5. Billah M., Adom K., Osae M., Jiang J. et Du J. (2014). Evaluation of the Great Fruit Fly Bait (GFFB) against fruit flies in Two Mango-Production zones in Ghana. Guide, 2p.

6. Mamadou T.S.D., Mahmoud C., Mastan Y.D.,Alpha S.B., André M.P., Lanciné T. (2003). Vers une lutte contre la cercosporiose des agrumes en Guinée. In : Effectiveness of Spinosad Bait Sprays (GF-120) in Controlling Mango-Infesting Fruit Flies (Diptera: Tephritidae),

7. Mwatawala M.W., White I. M., Maerere A. P., Senkondo F. J., De Meyer M. (2004). A new invasive Bactrocera species (Diptera : Tephritidae) in Tanzania. African Entomology, 12 (1): 154-156.

8. Mwatawala M. W., De Meyer M., Makundi R. H., Maerere P. A. (2006). Biodivresity of fruit flies (Diptera: Tephritidae) in orchards in different agro-écological zones of the Morogoro, Tanzania. Fruits, 61 (5): 321-332.

9. N’Dépo O.R., Hala N.F., Allou K., Aboua L. R., Kouassi K. P., Vayssières J. F., De Meyer M. (2009). Abundance of fruit flies in 
fruit producing areas of Ivory Coast: population dynamics of Bactrocera invadens (Diptera: Tephritidae). Fruits, 64 (5) : 313324.

10. N'dépo O. R. (2006). Inventaire des mouches des fruits (Diptera : Tephritidae) dans les stations expérimentales d'Azaguié et de Yamoussoukro (Côte-d'Ivoire). Mémoire D.E.A d'Entomologie Générale, Université de Cocody Abidjan (RCI), 59 p.

11. N'dépo O. R., Hala N. F., Gnago A., Allou K., Kouassi K. P., Vayssières J. F., et De Meyer M. (2010). Inventaire des mouches des fruits de trois régions Agro-écologiques et plantes-hôtes associées à l'espèce nouvelle Bactrocera (Bactrocera) invadens Drew et al. (Diptera : Tephritidae) en Côte d'Ivoire. European Journal of Scientific Research, 46 (1) : 6372.

12. Oukil S., Bues R., Toubon J. F. et Quilici S. (2002). Allozyme polymorphism in population of Ceratitis capitata from Algeria, the north western Mediterranean coast and Réunion Island. Fruits, 57 (3): 143-191

13. Quilici S., Duyck P. F., Rousse P., Courdon F., Simiand C., Franck A. (2005). La mouche de la pêche sur mangue, goyave etc. Phytoma. La Défense des Végétaux, $\mathrm{N}^{\circ} 584$ : 44-47.

14. Quilici S., Jeuffrault E, Ryckewaert P., Rolet A. (2006). Les mouches des légumes. Fiche technique programme régional de protection des végétaux, CIRAD, 4 p. http://www.prpv.org//, le 17/04/2007.

15. Rey J. Y., Thierno M. D., Vannière H., Christian D., Sidiki K., Morodjan S. (2004). La mangue en Afrique de l'ouest francophone : variété et composition variétale des vergers. Fruits, 59(3): 191208

16. Vayssières J. F., Fatogoma S., Moussa N. (2004). Inventory of species of fruit flies (Diptera: Tephritidae) linked to the mango tree in Mali and testing of rational control. Fruits, 59(1): 1-14.

17. Vayssières J-F., Georg G., Orphée L., Dossa P., Akponon C. (2005). A new Bactrocera species in Benin among fruit fly (Diptera: Tephritidae) species. Fruits, 60 (6): 371-377.

18. Vayssières, J-F., Sanogo F., et Noussourou M. (2007). In Effectiveness of Spinosad Bait Sprays (GF-120) in Controlling Mango-Infesting Flies (Diptera: Tephritidae). Fruits 62: 329-341

19. Vayssières, JF., Korie S.,Coulibaly O., Temple L. et Boueyi S. P. (2008). The mangoes tree in nortthem Benin: cultivar inventory, yield assessment, infested stages and loss due to fruit flies (Diptera: Tephritidae) Fruits, 63: 1-8. 
20. Vayssières J-F., Korie S., Coulibaly O., Melle C.V., Temple L., Arinloye D. (2009). The mango tree in central and northern Benin: damage caused by fruit flies (Diptera: Tephritidae) and computation of economic injury level. Fruits, $64: 207-220$

21. Vasquez J., Delgado C., Couturier G. et Matile D. F. (2002). Les insectes nuisibles au goyavier (Psidium guayava L.: Myrtaceae) en Amazonie péruvienne. Fruits, 57 (5-6): 267-351.

22. White I.M., Elson-Harris M. (1992). Fruit flies of economic significance: their identification and bionomics. Cab Int., Aciar, Redwood Press, Melksham, UK, 601p. 